\title{
Grasp Sensing for Human-Computer Interaction
}

\author{
Raphael Wimmer \\ University of Munich \\ Amalienstr. 17, 80333 Munich, Germany \\ raphael.wimmer@ifi.lmu.de
}

\begin{abstract}
The way we grasp an object depends on several factors, e.g. the intended goal or the hand's anatomy. Therefore, a grasp can convey meaningful information about its context. Inferring these factors from a grasp allows us to enhance interaction with grasp-sensitive objects. This paper highlights an grasp as an important source of meaningful context for human-computer interaction and gives an overview of prior work from other disciplines. This paper offers a basis and framework for further research and discussion by proposing a descriptive model of meaning in grasps The GRASP model combines five factors that determine how an object is grasped: goal, relationship between user and object, anatomy, setting, and properties of the object. The model is validated both from an epistemological perspective and by applying it to scenarios from related work.
\end{abstract}

\section{ACM Classification Keywords}

H.5.2 Information Interfaces and Presentation:

User Interfaces;

H.1.2 User/Machine Systems: Human factors

\section{General Terms}

Human Factors, Theory

\section{Author Keywords}

grasp recognition, tangible user interface, grasp, meaning

\section{INTRODUCTION}

During evolution, humans gained the unique ability to grasp tools and apply them skillfully. Nowadays, a multitude of versatile or specialized tools extend our manual abilities. Oftentimes, Human-Computer Interaction (HCI) also relies on our ability to grasp objects - be it a computer mouse, a tangible user interface, or a mobile phone. Given that we spend a reasonable amount of time grasping objects while using them, it seems straightforward to use the contact between device and hand for transmitting information to the user. Haptic feedback sends short cues to users via actua-

Permission to make digital or hard copies of all or part of this work for personal or classroom use is granted without fee provided that copies are not made or distributed for profit or commercial advantage and that copies bear this notice and the full citation on the first page. To copy otherwise, or republish, to post on servers or to redistribute to lists, requires prior specific permission and/or a fee.

TEI'11, January 22-26, 2011, Funchal, Portugal.

Copyright 2011 ACM 978-1-4503-0478-8/11/01...\$10.00.

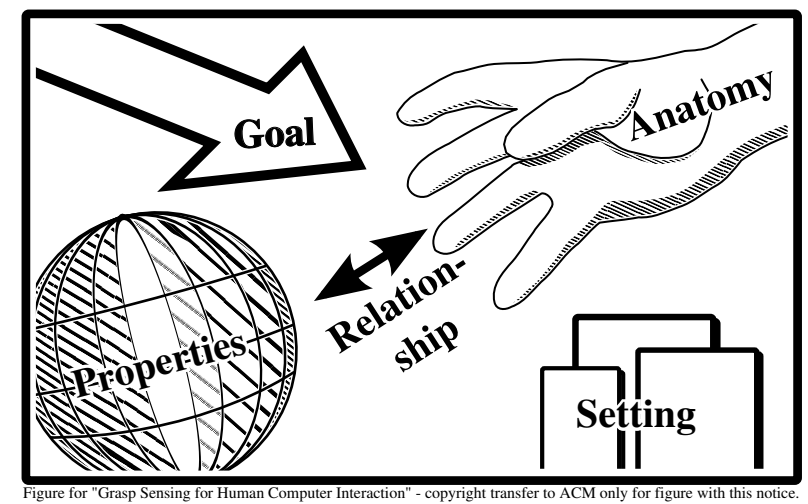

Figure 1. The way we grasp an object conveys meaningful information. Several factors determine a grasp. Our GRASP model groups them into five general factors: Goal of the grasp, Relationship between user and object (e.g. knowing that an object is very expensive), Anatomy of the user (including neuropsychological processes), Setting (i.e. environment in which the grasp takes place), and Properties of the object. Factors were chosen to represent orthogonal types of meaning

tors that stimulate tactile receptors in the skin. Such cues and status information can also be represented as changes in weight distribution or shape [9, 10]. However, a grasp actually allows bi-directional data transfer. The way we grasp an object also conveys information about us, our goals, and other aspects. Inferring these aspects from the grasp can allow us to enhance interaction with graspable user interfaces. The way we grasp an object is determined in part by what we want to do with it. Knowing the intended goal of a grasp, a grasp-sensitive object could support this goal. For example, a mobile phone would infer from your grasp that you want to call someone - and would present the call list. A bottle might know that you want to drink from it, and open itself - because you grasped it near its center of gravity. And a power drill might refuse to power on because you are not holding it safely enough. With each grasp we convey information - and given that we grasp objects all the day, this is a lot of information we produce. Currently, almost all of this information is ignored, however. The aim of this paper is to raise awareness to grasping as an important source of meaningful context for human-computer interaction, give an overview of existing work from other disciplines, and provide a basis for further research by proposing a descriptive model of meaning in grasps (see Figure 1).

This paper is structured as follows: In the next section I give an overview of related work from robotics, medicine and 
neuropsychology. Then I discuss technical foundations of grasp sensing, presenting a three-stage process for capturing, identifying, and interpreting grasps. Afterwards, I present a descriptive model of the meaning of grasps and discuss its validity, applications, and limitations.

\section{RELATED WORK}

Human's ability to purposefully grasp arbitrary objects has been subject of intensive research in different disciplines. The biomechanical properties of the human hand are subject of medical research [20]. In neuropsychology, researchers investigate how grasps are planned and controlled [6]. In robotics, researchers investigate how robotic hands may reliably grasp arbitrary objects [25]. MacKenzie and Iberall [17] give a comprehensive but slightly dated overview of research in these fields.

\section{Definitions}

In this paper, a grasp is defined as a "firm hold or grip" [1]. Accordingly, grasping means performing a grasp. Stable grasps can be divided into two different types [21]: Force closure - whereby the object is held by opposing fingers exerting pressure against the object's surface.

Form closure - whereby the object is enclosed by the hand so that it cannot move even if no force is exerted by the fingers. Often the terms grasping and prehension are used interchangeably [17]. For others, prehension is the coordinated act of reaching for an object and grasping it [29]. Kang and Ikeuchi state that a grasping task is composed of pre-grasp phase, static grasp phase, and manipulation phase [13].

\section{Classifications of Grasps}

Several classifications of grasps have been proposed over time. Schlesinger [24] classified grasps based on the shapes of objects they were applied to. Napier [20] proposed a simple classification of grasps into power grips, which generally involve the palm, and precision grips, which only employ fingers and thumb. Cutkosky and Wright [7] use a tree hierarchy for classifying grasps, beginning with Napier's power and precision grasps. Kang and Ikeuchi [13] recognized that the aforementioned models lack a formal description of the grasps which makes them unsuitable for automated grasp recognition. They proposed the contact web, as a formal classification of grasps that is based on contact points between object and fingers. Feix at al. condensed 147 grasp descriptions found in a review of 17 publications into 33 unique grasps [8]. These were classified based on involved fingers, Napier's distinction, opposition type, and thumb position.

\section{MEANING IN A GRASP}

Existing classifications focus on defining groups of visually or functionally distinctive grasps. However, a multitude of factors contribute to a grasp which are not considered in these classifications.

Iberall and MacKenzie offer an abstract model of grasping $[12,17]$. They describe grasping as a black box with a goal and an object as inputs and a specific grasp as output. Several additional constraints are given that affect the outcome, like the placement and orientation of the object.
However, MacKenzie and Iberall do not explain the formal difference between inputs and constraints. These constraints are grouped by MacKenzie and Iberall [17] as follows:

- High Level: Social/Cultural, Motivational, Informational, Functional

- Physical: Object Properties, Biomechanical/Mechanical

- Sensorimotor: Neural, Perceptual, Anatomical/Physiological, Evolutionary/Developmental

However, for HCI the question is not how a grasp should look like for a certain combination of object and goal (humans are quite good at figuring it out themselves). Instead it would be useful to know what a grasp actually means. That is, what can a grasp tell us about the context it is happening in, and what can we do with this context intformation. For a certain grasp - which we can capture by different means we want to know which factors contributed to it.

\section{GRASP SENSING}

This section explains why it is acceptable and helpful to only capture stable grasps, and defines three interpretative steps for grasp-sensing systems: capturing the grasp, identifying it, and interpreting it (Figure 2).

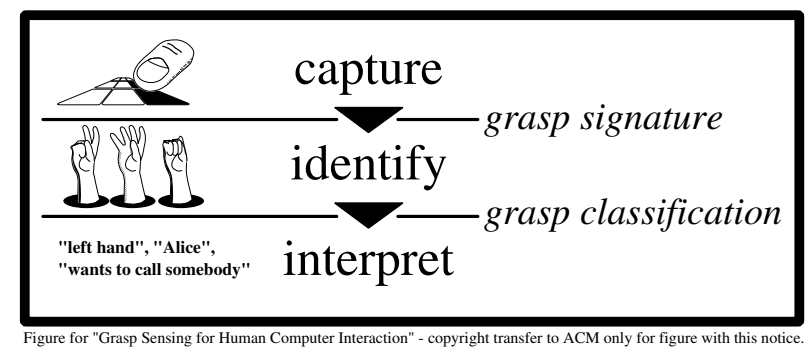

Figure 2. General workflow for grasp sensing: sensors capture a grasp signature which gets mapped to a specific group of grasps. Finally, interpreting the grasp generates meaningful context.

\section{An important simplification}

Grasping is a complex action. Depending on the point of view it also includes initial reaching movements and exploratory adjustments until we find a stable grasp. Sometimes, we move through a number of different grasps, even involving different hands - e.g. when picking up an object and putting it somewhere else. Complex hand movements are needed to transform one stable grasp into another. However, in the following I will use the term grasp to refer only to single, stable, static grasps, performed by a human hand. This has a formal and a technical reason:

- a series of grasps can be broken up into a number of stable grasps. The transitional hand movements between those stable grasps primarily depend on the previous and next grasp. Therefore, these transitions add only very little information about the meaning of a grasp ${ }^{1}$. Excluding those

\footnotetext{
${ }^{1}$ An exception would be a slippery object that is held in a formclosure grasp. The (stable) grasp does not need to adress the slippery surface. However, any transitional hand movement would need to take this into account.
} 
transitions from our analysis removes much complexity while forfeiting only little information.

- the aforementioned complexity of transitional movements makes them hard to interpret using heuristics or machinelearning. Concentrating on stable grasps makes it much easier to extract meaningful patterns.

\section{Step 1: Capturing Grasps}

Grasps can be captured by either capturing the grasping hand or by equipping the object with a grasp-sensitive surface. Both approaches have unique advantages and disadvantages. For everyday use, objects with grasp-sensitive surfaces are probably more convenient and socially acceptable, however.

\section{Instrumenting the User}

For tracking fingers and hand, often an optical or magnetical tracking system is used. A marker or receiver needs to be attached to each finger. This method allows for a highresolution capture of hand pose and position. It does not provide any information about contact forces between finger and object. Therefore it can not detect whether a finger is touching the object or not. Such tracking systems need to instrument the user. Additionally, capturing grasps works only within the tracking infrastructure. Therefore such systems are of limited use for real-world applications. However, they are very flexible and therefore well suited for laboratory studies. External tracking systems also provide a highresolution ground truth for other technologies.

Instead of using markers for defining finger joints, some approaches use computer vision techniques for an image of the hand pose to a grasp [31]. Another approach that does not require a tracking infrastructure are data gloves. These are thin gloves - often with a mesh surface on the palmar side - that are equipped with strain sensors. These sensors allow capturing each finger joint's angle. From these, a hand pose can be derived [11]. These gloves are less obtrusive than tracking markers. However, they limit tactile perception. Force-sensing with gloves is difficult, as force sensors attached to the fingertips obviously result in greatly reduced tactile perception. An alternative way of determining grasp forces is to capture images of the finger nail and interpret changes in blood flow that can be observed there [19].

As the fingers are controlled by muscles in the forearm, some information about the hand pose can also be gained from measuring changes in these muscles or the wrist shape [22]. Another option is not to capture grasps but only detect which object is held. This can be done by attaching RFID tags to all objects and strapping an RFID reader to the user's wrist [4]. This method does not provide any information about the actual grasp, however.

Instrumenting the users allows for easily capturing grasps for a multitude of object shapes. The objects need not be modified. However, putting markers on hands would severly impede everyday manual tasks. Additionally, in most applications the object itself shall react to a grasp. Therefore, the grasp information would first need to be transferred to the object - either wirelessly or by electrical coupling [34]. This would require a standardized communications protocol for grasp information.

\section{Grasp-sensitive Surfaces}

Grasp information can also be captured by the grasped object itself. To this end, the object needs to be equipped with a grasp-sensitive surface that can capture the contact area between object and hand. Grasp-sensitive surfaces require more engineering effort than external tracking solutions and usually do not offer identification of single fingers or digits. Implementing a grasp-sensitive surface is more difficult than implementing a touch-screen for two reasons:

Shape Sensing. Instead of single or multiple touch points, a grasp-sensitive surfaces should capture multiple complex shapes and possibly additional information like pressure. Therefore, common commercial touchscreen technologies like (ana$\log$ ) resistive[16] or self-capacitance [3] touch sensing cannot be used. Digital resistive [23] or mutual-capacitance technologies are better suited for capturing contact areas shapes. Additionally, the driver software needs to be able to represent complex shapes.

Sensor Shape. The sensing surface is usually non-planar and continuously wrapped around the whole object. While it is possible to embed only a few sensors at strategic positions within the surface[5,33], often large parts of the surface shall be made grasp-sensitive. This requires flexible or very small sensors. Additionally, a normal cartesian coordinate system - as used in displays and touch input - may not correctly represent the sensing surface. Possible alternatives are spherical coordinate systems[15] or a relative mapping[32].

In research prototypes a multitude of sensor technologies are used for making surfaces grasp-sensitive. Often researchers build their own capacitive sensors [14, 27, 33]. Other prototypes use resistive pressure sensors [15, 30], impendance sensors [18] or optical sensors [5, 32]. A comparison of the inherent properties of the different types of sensors can be found in [32].

Capturing a grasp results in a grasp signature, a digital representation of contact points or digit positions.

\section{Step 2: Identifying Grasps}

Once a grasp signature has been captured, the system needs to derive the grasp that caused this signature [13]. This is commonly done by mapping the signature to a certain category of grasps, either using heuristics or machine learning. The classification algorithm to be used depends both on the format of the sensor data and the type of information that is needed in the interpretation stage. Commonly used classifieres include support vector machines [14, 26], Bayesian classifiers [14, 26], and Hidden-Markov-Models [27]. Grasp categories can be defined formally, using e.g. one of the models described above. More often, grasp categories are defined by example. To this end, a machinelearning classifier is trained with grasp signatures of grasps the developer deems similar. Without a set of formally defined grasps, however, it is not possible to compare different classifiers and sensing technologies. In several cases, grasps with completely different signatures should actually be interpreted as the same grasp. For example, when grasping a uniform sphere, the grasp signature greatly depends on the rotation of the sphere. In these cases, an appropri- 
ate mapping needs to be found that ignores certain features of a grasp. Wimmer et al. [33] recognize different types of left-handed and right-handed grasps using slightly modified heuristics for both. Kry et al. [15] use spherical harmonics for rotation-invariant grasp classifications.

Classifying a grasp signature results in a grasp classification - for example a list of grasps that might have generated the grasp signature.

\section{Step 3: Interpreting Grasps}

The final step in graps sensing is to determine what a captured and classified grasp actually means. What is to be considered meaningful depends on the application. For authentication purposes [30], it is relevant whether the grasp belongs to a certain category - whereby different categories represent different users. When using grasps to switch an object between different modes [27], the mapping from a certain grasp category to a mode provides meaning. However, existing grasp-sensitive systems capture only one meaningful aspect of a grasp (see below), ignoring the other aspects. While such systems attribute a certain meaning to a grasp, the actual meaning of a grasp is ultimately defined by the user. This leads to unexpected behavior if the system attributes a meaning to a grasp that the user did not intend. For example, a grasp-sensitive mobile phone might interpret a certain grasp as the intention to switch to camera mode [14]. However, the user might just hold the phone this way for a variety of other reasons - and would be annoyed if the phone suddenly changed into a different mode.

Insofar it seems advisable to try to capture as much information about the meaning of a grasp as possible. In the following section I look at meaningful factors that influence which grasp is chosen.

\section{GRASP - A MODEL OF MEANING IN GRASPS}

As stated above, a model of meaning within a grasp is needed. The model I present here is based on the model by MacKenzie and Iberall that is described in the second section. The new model considers the constraints and inputs they described and re-organizes them into meaningful factors. An important simplification is abstracting the user, hiding much of the neuropsychological and anatomical complexity. This probably does not remove meaningful information as most statements we might be able to make about such sub-factors will not help us in improving the user interface. Being aware of this abstraction allows us to replace it with a more fine-grained representation, if needed.

In the following I present GRASP, a model of human grasping that describes five meaningful factors. These factors all influence which grasp a user applies, and each of them represents a different group of meaningful information. Figure 3 illustrates these factors. All factors are independent of each other. The validity of this model is discussed in the next section.

\section{Goal}

A very important factor determining how to grasp a given object is the goal that is to be achieved by the grasp. Goals are all factors that cause a grasp to be initiated. Goals can be divided into sub-goals. As goals are essentially meaning, they are discussed here in more detail than the other factors. I propose grouping goals by two dimensions: implicit vs. explicit grasps and primary vs. supportive grasps. Table 1 shows examples for each combination of implicit/explicit and primary/supportive grasps.

\begin{tabular}{l|l|l} 
& \multicolumn{1}{|c|}{ implicit } & explicit \\
\hline primary & $\begin{array}{l}\text { user picks up mo- } \\
\text { bile phone }\end{array}$ & $\begin{array}{l}\text { user holds phone } \\
\text { horizontally to in- } \\
\text { voke photo mode }\end{array}$ \\
\hline supportive & $\begin{array}{l}\text { user holds phone } \\
\text { while typing on } \\
\text { touchscreen with } \\
\text { other hand }\end{array}$ & $\begin{array}{l}\text { user holds phone, } \\
\text { scrolling a map } \\
\text { with the other } \\
\text { hand, zooming } \\
\text { by squeezing the } \\
\text { phone }\end{array}$
\end{tabular}

Table 1. Examples illustrating the distinction between implicit and explicit grasp interaction, respectively primary and supportive grasps.

Usually, a grasp is implicit, meaning that its goal is to manipulate an object. Implicit grasps are similar for graspsensitive and non-grasp-sensitive objects. Explicit grasps are conducted primarily in order to trigger a certain effect in a grasp-sensitive object. For example, a user might authenticate himself to the device using a certain grasp, or switch the mode of an object by holding it in a certain way. Likewise, there is a difference in the user's intention for primary and supportive grasps. A primary grasp is a grasp that is executed with the intention to manipulate the grasped object. For example, a primary grasp is used for moving a computer mouse. A supportive grasp serves to fixate or position an object in order to interact with it using e.g. the other hand or fingers that are not involved in grasping. For example, holding a mobile phone while typing a short message is a supportive grasp. These distinctions are not random but are needed to judge the amount of meaning gained from a grasp. An explicit grasp always conveys information about the user's intention. It can and should always be seen as meaningful. The user expects a reaction. An implicit grasp may convey some information about the user's intention. However, this intention is not necessarily aimed at interacting with the object. A user might just want to put away an object and would be annoyed if it reacts to the grasp. Therefore, determining whether a grasp is implicit or explicit is necessary for interpreting the grasp's meaning. Likewise, a supportive grasp conveys less meaning than a primary grasp as the main focus of the interaction lies not on the grasp but on the action that is supported by it. Additionally, a supportive grasp is restricted by the requirements of the main interaction and usually can not be modified as easily as a primary grasp. One might use this distinction to assign different meanings to a grasp. For example, when holding a mobile phone to the ear (primary grasp), different grasps can launch actions. When holding the phone while interacting with its touchscreen using the other hand, different supportive grasps might only switch between modes or adjust viewport properties. 


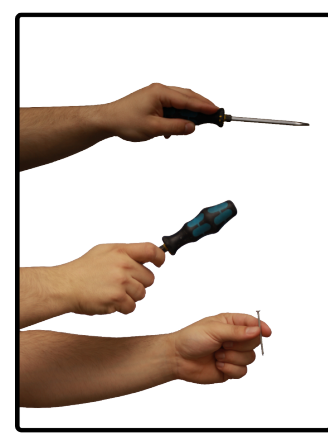

Goal

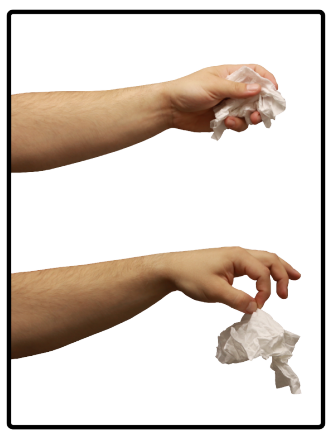

Relationship

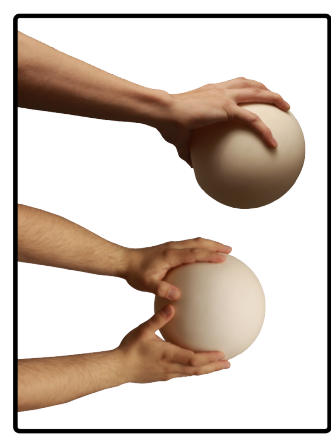

Anatomy

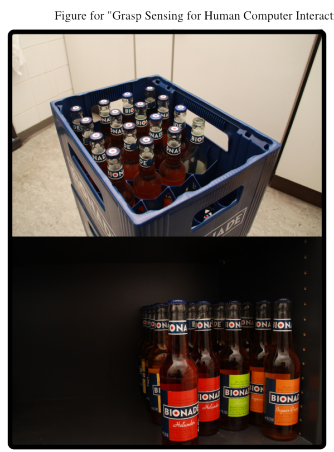

Setting

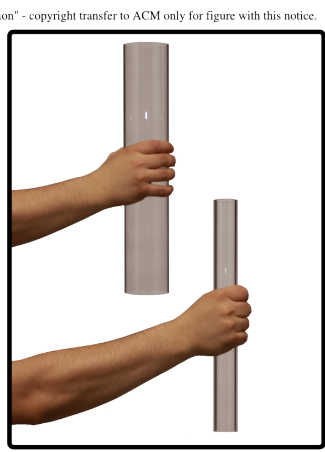

Properties

Figure 3. Examples for each of the meaningful factors that contribute to a grasp. A user might hold a screwdriver differently, depending on the Goal he wants to achieve with it. The mental Relationship to an object determines how we grasp it. In the illustrated case, a paper towel that belongs to oneself is held differently than one found on the floor. Depending on our Anatomy we need to apply different grasps to achieve the same effect. Due to different Settings, a bottle in a shelf has to be grasped differently than a bottle in a crate. The Properties of an object, for example its size, have a major influence on the grasp to be used.

\section{Relationship}

Feelings like disgust, fear, or anger can also influence the way we grasp. Relationship includes all non-physical factors that apply to a combination of user and object. The relationship between user and object can be different for every user-object combination. For example, many people seem to find a used paper towel disgusting. They would only pick it up with a pinching grasp, trying to avoid contact with anything attached to it. However, if the used paper towel belongs to oneself, one might just pick it up like any arbitrary piece of paper. Unlike anatomical properties, the relationship can also change over time, like when someone overcomes a fear of spiders and is able to touch one. How a feeling changes a grasp is also determined by the knowledge about an object. An angry person might unconsciously grasp the steering wheel more tightly but would not do the same with an egg. These effects are not solely properties of the grasping person or the object but depend on the combination of grasping person and grasped object. Relationship probably has little impact on most grasps. However, this factor has not been explored in detail so far.

\section{Anatomy}

Not every hand grasps the same way. Anatomy includes all factors that are inherent to the grasping person's body. Obviously, differences in palm size, finger length, or finger count result in slightly different grasps. Additionally, differences in sensorimotor control of grasps also causes different grasps. Differences in people's grasps might be used for automatically identifying the person grasping an object. A power tool might also detect insufficient grasp forces for a stable grasp and warn users that they have to hold it more tightly.

\section{Setting}

The environment within which a grasp takes place also affects its outcome. Setting includes all factors that pertain to the environment in which a grasp takes place, i.e. all physical factors that are independent of user and object. For exam- ple, the grasp used for pulling a bottle out of a crate differs from the one used for picking the bottle from a shelf. Additional environmental factors might include lighting conditions, space constraints, or temperature.

\section{Properties}

The properties of the object to be grasped naturally have a huge influence on the grasp to be used. Properties are all factors that are intrinsic to the object. This includes shape, size, surface texture, weight and weight distribution, or surface temperature.

\section{VERIFYING GRASP}

This section explains why GRASP is indeed a model, and that it fulfils formal requirements of a model. Finally, several examples highlight how GRASP can be applied.

\section{Formal Validity}

There are a variety of views what a model actually is. BailerJones offers the definition[2]: "A model is an interpretative description of a phenomenon that facilitates access to that phenomenon.". GRASP is a model according to this definition as it describes a certain aspect (the meaning) of a phenomenon (a grasp). However, what are the criteria for a good model? I have chosen Vaandrager's definition of a good model [28]. While it pertains to models in computer system analysis, it is a concrete checklist that with plausible requirements. Vaandrager lists seven properties of a good model but notes that some of these contradict each other sometimes. These properties have been rephrased in the following to keep them succinct.

According to Vaandrager, a good model should:

...have a clearly defined object of modeling. GRASP pertains to static human grasps.

...have a clearly specified purpose.

GRASP defines meaningful factors contributing to a grasp 
...clearly show the links between model and empirical world.

each factor is clearly tied either to a clear concept (Goal, Relationship) or a physical object (Anatomy, Setting, Properties)

...be truthful, i.e. correctly represent relevant properties of the object of modelling.

As a model is a simplification of a complex matter, there is not only one correct model. Additionally, a model inherently oversimplifies certain aspects of a phenomenon. Therefore, a model can not be completely truthful (as acknowledged by Vaandrager). Additionally, meaning is a property that is attributed to a phenomen by humans. Therefore it is hard to tell if our distinction of meaningful factors in a grasp is correct. However, we can look at the meaning other researchers attribute to a grasp and see whether it matches to the factors we defined:

- Veldhuis et al.[30] solely try to derive the Anatomy of a user from a grasp.

- Taylor and Bove[27] solely try to derive an explicit Goal from a grasp.

- Kim et al.[14] solely try to derive an explicit Goal from a grasp.

- Wimmer and Boring[33] solely try to derive an implicit Goal from a grasp. In the two cases without a grasp, a Setting is guessed from the sensor data.

- SpherUID - a yet unpublished project - tries to determine the user's position in relation to the object (Setting) from a grasp.

- Kry et al.[15] solely tries to derive an explicit Goal from the grasp.

Therefore our model seems to correctly identify at least Goal, Anatomy, and Setting as meaningful factors of a grasp. I do not know of any application deriving Relationship or Properties from a grasp, however.

...be as simple as possible - but not too simple.

For GRASP this would mean that none of factors can be omitted. Otherwise, the model could be simplified. That is, for each factor at least one situation exists where the grasp is primarily determined by the factor. Examples of such situations are given in the descriptions of the factors. The requirement also indicates that none of the factors may be partially covered by another (i.e. they should be orthogonal). Otherwise the model would need to be more granular and therefore more complex. A goal can be defined without knowing any of the other factors. Therefore it may not contain sub-factors also found in the other factors. Relationship explicitly covers sub-factors that are independent of the user's and the object's physical properties. The Relationship also only pertains to a certain user-object combination and is therefore independent of goal and setting. Anatomy, setting and properties pertain to inherent properties of user, environment, and object. Each of them is a clearly defined physical object. Thus, they share no common sub-factors with each other or the other factors. ...be extensible and reusable.

GRASP is intended as a working draft. Therefore, it is also intended to be extended. For example, GRASP could be used as a basis for a predictive model. I discuss this idea in the Future Work section.

\section{...allow interoperability with related models.}

GRASP shares the the concepts of Goal and Properties with other models. Anatomy combines multiple factors from other models. Setting - while not explicitly named in the other models - seems to be compatible to them. Relationship is a newly introduced factor. It is not yet completely clear how it relates to other models.

Overall, using above criteria, GRASP seems to be a useful and robust model of meaning in grasps.

\section{Practical Applications}

In the following, the GRASP model is exemplarily applied to two different grasp-sensitive applications which have been proposed in related work. The goal is to identify which issues need to be considered when actually implementing such applications

Both Taylor and Bove [27] and Kim et al [14] propose to use grasp recognition for mode switching. For example, a mobile phone might switch between camera mode (two-handed grasp) and short-message typing mode (one-handed, thumb on keypad), depending on the way it is being held. The GRASP model can be used to highlight which factors of a grasp were investigated in the prototype, and which importance these factors have in real-life scenarios:

Goal: recognized: switch mode (implicit, might become explicit with regular use)

Relationship: ignored, in real-life scenarios probably mostly constant, as a mobile phone is a personal device

Anatomy: varied, in real-life scenarios probably mostly constant, as a mobile phone is a personal device

Setting: assumed constant (lab study), in real-life scenarios setting changes constantly

Properties: assumed constant, prototype does not change properties

GRASP also allows a structured analysis of the design space, revealing a number of challenges faced when implementing mode-switching functionality for mobile phones:

- Users might get accustomed to the mode-switching behavior. It should be checked - e.g. with a long-term study whether this affects their use. Persons using such a phone for the first time might get irritated.

- The phone should achieve good grasp recognition accuracy for a single user but should not completely fail if another person uses it (both systems were trained using grasps from multiple persons).

- It should be tested how different settings affect recognition performance.

- It should be tested how well the system performs if the phone's weight distribution or shape change $[10,9]$. 
Veldhuis et al [30] present a a prototype of a grasp-sensitive gun. It recognizes its user based on the pressure pattern of the grasp. Only authorized users shall be able to use the gun. Like before, GRASP can be used to investigate which meaningful factors of a grasp are considered by this prototype:

Goal: assumed constant, defined as "fire gun" (implicit)), in real-life scenarios probably mostly constant

Relationship: ignored, in real-life scenarios probably mostly constant, as a gun is a personal device

Anatomy: recognized

Setting: controlled (lab study), will vary greatly in real-life scenarios

Properties: constant, prototype does not change properties

Similarly, a structured analysis of the design space reveals a number of challenges:

- Users might have different goals when grasping the gun, e.g. cleaning it. The system should not treat this as an unauthorized attempt to use the gun.

- A gun may be used in various different settings, e.g. while running, or in tight spaces. The system needs to correctly identify authorized users in all these conditions.

- Sweat or rain might make the gun's handle slippery, requiring the user to grasp differently.

In summary, the GRASP model proposed in this paper can be used for categorizing grasp-sensitive objects, exploring the design space of grasp-sensing applications, and analyzing challenges when implementing grasp-sensitive user interfaces.

\section{Limitations}

GRASP is a descriptive model of meaning in grasps. As such it is based on a subjective concept. Different persons and cultures might prefer other groupings. GRASP is also limited to meaningful interaction. Thus it is not necessarily suited for other domains. While I have tried to show that this model is formally valid and can be useful for reseachers and designers, ony time will tell whether this model is actually helpful. Finally, I am not yet certain whether Relationship really is an own factor. One might als group it with Anatomy into a Human factor. Some might argue that Relationship is a actually just a modifier to a Goal. In the end, it might depend on whether it is useful to define Relationship as an own factor.

\section{CONCLUSION AND FUTURE WORK}

In this paper I have argued that grasp sensing is an important source of meaningful information for human-computer interaction. I have given an overview of existing work from other disciplines and have shown that existing models are not sufficient for describing grasps in the context of humancomputer interaction. Therefore, I propose a descriptive model of meaning in grasps. This model is supported by examples and an epistemological analysis. GRASP is intended as a working model. The main goal for GRASP is to serve as a vocabulary and basis for discussing meaning in grasps and grasp interaction utilizing this meaning. This model may also help in structuring research, e.g. by highlighting interesting areas of further research. It can also be used to determine which factors need to be controlled in a user study. An important long-term goal would be to automatically extract all factors contributing to a grasp from just the signature of the grasp. For this, a classifying model would be helpful, associating certain quantitative features of a grasp with the meaningful factors contributing to it.

\section{REFERENCES}

1. The American Heritage Dictionary of the English Language, Fourth Edition. Houghton Mifflin, 2009.

2. D. Bailer-Jones. Scientific models in philosophy of science. Univ of Pittsburgh Pr, 2009.

3. G. Barrett and R. Omote. Projected-Capacitive Touch Technology. Information Display, 26(3):16-21, 2010.

4. E. Berlin, J. Liu, K. van Laerhoven, and B. Schiele. Coming to grips with the objects we grasp: detecting interactions with efficient wrist-worn sensors. In TEI '10: Proceedings of the fourth international conference on Tangible, embedded, and embodied interaction, pages 57-64, New York, NY, USA, 2010. ACM.

5. A. Butler, S. Izadi, and S. Hodges. Sidesight: multi-"touch" interaction around small devices. In Proceedings of UIST '08, pages 201-204, New York, NY, USA, 2008. ACM.

6. U. Castiello. The neuroscience of grasping. Nature Reviews Neuroscience, 6(9):726-736, 2005.

7. M. Cutkosky. On grasp choice, grasp models, and the design of hands for manufacturing tasks. IEEE Transactions on Robotics and Automation, 5(3):269-279, 1989.

8. T. Feix, R. Pawlik, H.-B. Schmiedmayer, J. Romero, and D. Kragic. The generation of a comprehensive grasp taxonomy. Technical report, 2009.

9. F. Hemmert, S. Hamann, M. Löwe, A. Wohlauf, and G. Joost. Weight-Shifting Mobiles: One-Dimensional Gravitational Displays in Mobile Phones. Adjunct Proceedings of UIST, 9, 2009.

10. F. Hemmert, G. Joost, A. Knörig, and R. Wettach. Dynamic knobs: shape change as a means of interaction on a mobile phone. In CHI'08 extended abstracts, pages 2309-2314. ACM, 2008.

11. G. Heumer, H. Amor, M. Weber, and B. Jung. Grasp recognition with uncalibrated data gloves-A comparison of classification methods. In IEEE Virtual Reality Conference, 2007. VR'07, pages 19-26, 2007.

12. T. Iberall and C. MacKenzie. Opposition space and human prehension. In Dextrous robot hands, pages 32-54. Springer-Verlag New York, Inc., 1990.

13. S. Kang and K. Ikeuchi. Grasp recognition using the contact web. In Proc. IEEE/RSJ Int. Conf. on Int. Robots and Sys., Raleigh, NC, 1992. 
14. K. Kim, W. Chang, S. Cho, J. Shim, H. Lee, J. Park, Y. Lee, and S. Kim. Hand Grip Pattern Recognition for Mobile User Interfaces. In In Proc. AAAI '06, volume 21, page 1789, 2006.

15. P. G. Kry and D. K. Pai. Grasp recognition and manipulation with the tango. In International Symposium on Experimental Robotics, volume 10. Springer, 2006.

16. J. Loviscach. Two-finger input with a standard touch screen. In Proceedings of the 20th annual ACM symposium on User interface software and technology, page 172. ACM, 2007.

17. C. MacKenzie and T. Iberall. The grasping hand. North Holland, 1994.

18. J. Mäntyjärvi, K. Nybergh, J. Himberg, and K. Hjelt. Touch Detection System for Mobile Terminals. In Proceedings of MobileHCI '05. Springer, 2004.

19. S. Mascaro and H. Asada. Measurement of finger posture and three-axis fingertip touch force using fingernail sensors. IEEE Transactions on Robotics and Automation, 20(1):26-35, 2004.

20. J. Napier. The prehensile movements of the human hand. Journal of Bone \& Joint Surgery, British Volume, 38(4):902, 1956.

21. J. Ponce, S. Sullivan, A. Sudsang, J. Boissonnat, and J. Merlet. On computing four-finger equilibrium and force-closure grasps of polyhedral objects. International Journal of Robotics Research, 16(1):11-46, 1997.

22. J. Rekimoto. Gesturewrist and gesturepad: Unobtrusive wearable interaction devices, 2001.

23. I. Rosenberg and K. Perlin. The UnMousePad: an interpolating multi-touch force-sensing input pad. $A C M$ Transactions on Graphics (TOG), 28(3):65, 2009.

24. G. Schlesinger. Der Mechanische Aufbau der Kunstlichen Glieder. Ersatzglieder und Arbeitshilfen, part II, 1919.
25. K. Shimoga. Robot grasp synthesis algorithms: A survey. The International Journal of Robotics Research, 15(3):230, 1996.

26. B. T. Taylor and M. V. Bove. The bar of soap: a grasp recognition system implemented in a multi-functional handheld device. In CHI '08: $\mathrm{CHI}$ '08 extended abstracts on Human factors in computing systems, pages 3459-3464, New York, NY, USA, 2008. ACM.

27. B. T. Taylor and M. V. Bove. Graspables: grasp-recognition as a user interface. In In Proc. CHI '09, pages 917-926, New York, NY, USA, 2009. ACM.

28. F. Vaandrager. What is a good model? http://www.cs.ru.nl/ fvaan/PV/ what_is_a_good_model.html, 2010.

29. C. van de Kamp and F. Zaal. Prehension is really reaching and grasping. Experimental Brain Research, 182(1):27-34, 2007.

30. R. N. J. Veldhuis, A. M. Bazen, J. A. Kauffman, and P. H. Hartel. Biometric verification based on grip-pattern recognition. In E. J. Delp and P. W. Wong, editors, Security, Steganography, and Watermarking of Multimedia Contents, volume 5306 of Proceedings of SPIE, pages 634-641. SPIE, 2004.

31. R. Y. Wang and J. Popović. Real-time hand-tracking with a color glove. ACM Transactions on Graphics, 28(3), 2009.

32. R. Wimmer. FlyEye: Grasp-Sensitive Surfaces Using Optical Fiber. In In Proc. TEI '10, Jan. 2010.

33. R. Wimmer and S. Boring. HandSense - Discriminating Different Ways of Grasping and Holding a Tangible User Interface. In In Proc. TEI '09, February 2009.

34. T. Zimmerman. Personal Area Networks: Near-field intrabody communication. IBM SYSTEMS JOURNAL, 35(3\&4):610, 1996. 